\title{
Asociaciones y coproducción de servicios en municipios españoles ${ }^{1}$
}

\author{
Associations and public services provision in Spanish municipalities \\ Ma Jesús RodRíGUEz GARCíA \\ Universidad Pablo de Olavide \\ mjrodgar@upo.es
}

Recibido: 30/01/2012

Revisado: 08/03/2012

Aceptado: 16/04/2012

Disponible on line: 03/08/2012

\section{Resumen}

Como es sabido, las asociaciones constituyen un actor central en el pluralismo del bienestar, y, de hecho, juegan un papel clave en la prestación de servicios. Sin embargo, la implicación de las asociaciones en los procesos de provisión de servicios difiere según su especialización en el activismo político o en la prestación de servicios, junto con otros rasgos asociativos. La literatura al respecto también indica que la participación de las asociaciones en estos procesos genera cambios en su estructura interna (profesionalización y burocratización). En este artículo se pretende analizar este aspecto de la coproducción de servicios a través de una encuesta entre asociaciones en municipios españoles. También se analizará el contenido de la coproducción a través del tipo de servicios que se prestan, el sector de la población al que se destinan y el nivel de la administración pública con el que las asociaciones establecen acuerdos de coproducción. Los resultados evidencian la importancia de la especialización (política vs, prestación de servicios) como factor explicativo de la participación de las asociaciones en la coproducción de servicios, la influencia de este factor en procesos organizativos internos y la importancia del nivel de gobierno autonómico a la hora de establecer este tipo de colaboración entre administración pública y asociaciones.

Palabras clave: coproducción, participación, asociaciones, servicios públicos, pluralismo del bienestar.

\begin{abstract}
Associations are considered a key actor in the development of the welfare mix. In fact, they play an important role in providing public services. The involvement, however, of associations in the processes of service provision is different according to their specialization in political activism or service provision, as well as other associational traits. The literature also indicates that this involvement produces some changes in associations' internal structure (i.e. professionalization and bureaucratization). This paper seeks to show these phenomena among a representative survey of associations in Spanish cities. The content of co-production or service provision is also analyzed by studying sector, kind of services and the level of public administration with which associations establish agreements for services delivery. Main results show the importance of services provision specialization (policy vs. services provision) as a factor to explain the participation in co-production processes, the influence of this factor in internal organizational processes, and the importance of the regional level of government at the point of establishing these kinds of collaborative agreements between public administration and associations.
\end{abstract}

Keywords: Co-production, participation, associations, public services, welfare mix.

Referencia normalizada: Rodríguez García, M. J. (2012): «Asociaciones y coproducción de servicios en municipios españoles». Cuadernos de Trabajo Social, 25(2): 357-369.

Sumario: Introducción. 1. Asociaciones, servicios públicos y pluralismo de bienestar: la coproducción y sus consecuencias. 2. El análisis de la coproducción asociativa: preguntas y metodología. 3. Análisis y resultados. 4. Conclusiones. 5. Referencias bibliográficas.

${ }^{1}$ Los datos utilizados para este artículo proceden de la encuesta realizada entre asociaciones en ciudades españolas en el marco del Proyecto de Investigación Género, Participación y Sistemas Locales de Bienestar, financiado por el Instituto de la Mujer en la Convocatoria de Proyectos de I+D (2006). La investigación se desarrolló entre 2007 y 2010 y la encuesta se realizó en 2009. 


\section{Introducción}

Normalmente, el estudio de la participación de las asociaciones se centra en los procesos por los que trasmiten las demandas a las autoridades públicas; esto es, diversas actividades por las que tratan de influir en los procesos políticos que dan lugar a la aprobación, diseño y puesta en marcha de ciertas políticas y servicios, usando para ello un repertorio diverso: desde acciones de carácter más formal a otras informales y disruptivas. No obstante, cada vez en mayor medida suelen considerarse también como participación asociativa aquellos procesos en los que éstas establecen acuerdos de colaboración de distintas naturaleza con las administraciones para prestar servicios públicos, incorporándose así como un actor más en el nominado welfare mix o pluralismo del bienestar (EspingAndersen,1999; Navarro, 2001; Rodríguez Cabrero,1994, 1996, 2004, 2005 y 2006; Rodríguez y Codorniu,1996; Salamon y Anheier, 1992), no sólo mediante los servicios y actividades que ellas desarrollan, sino contribuyendo a la prestación de servicios de titularidad pública.

Concretamente, desde la perspectiva de los procesos que implican las políticas y servicios públicos, el primer tipo de actividades suponen el desarrollo de procesos de cooperación en la provisión de servicios públicos; esto es, la implicación de las asociaciones (u otros actores) en las decisiones en torno al diseño, la planificación y financiación de políticas y servicios públicos. El segundo tipo de actividades implica el desarrollo de procesos de coproducción de servicios públicos; esto es, su implicación en el momento en que estos son producidos y prestados directamente a la ciudadanía (Sharp, 1980; Whitaker, 1980).

En este artículo nos centraremos en el análisis de procesos de coproducción, esto es, acuerdos de diversa naturaleza (convenios, contratos, etc.) que se establecen con las entidades asociativas para la prestación de servicios de titularidad pública. No nos interesa su naturaleza jurídica, sino tres preguntas que suelen estar presentes en la literatura y el análisis existentes al respecto, sobre el papel de las asociaciones como agentes colaboradores de la Administración Pública respecto a la prestación de sus servicios. Estas cuestiones son: ¿cuáles son las características de las asociaciones que se involucran en esos procesos?, ¿qué consecuencias tienen sobre su dinámica y estructura organizativas?, ¿cuál es el contenidos de los acuerdos de coproducción? Esto es, los tipos de servicios que implican y con las administraciones públicas que se establecen.

En el primer apartado revisaremos brevemente algunas de las ideas desarrolladas en torno a estas preguntas. En el segundo presentaremos la metodología empleada para tratar de aportar evidencias, utilizando para ello una encuesta entre asociaciones de cinco ciudades españolas, desarrollada en el marco del proyecto Género, Participación y Sistemas Locales de Bienestar $^{2}$. En el tercer apartado presentamos los análisis realizados y en el cuarto y último apartados desarrollamos algunas de las conclusiones a las que hemos llegado.

\section{Asociaciones, servicios públicos y plura- lismo de bienestar: la coproducción y sus con- secuencias}

El fenómeno de la implicación de entidades asociativas en la producción de servicios de titularidad pública, mediante distintos tipos de acuerdos de colaboración, es un fenómeno bastante extendido, y se supone que se relaciona y tiene consecuencias sobre las actividades que desarrollan las asociaciones o sus dinámicas organizativas. Así, respecto al primer aspecto, el análisis de las pautas de participación de las entidades asociativas muestra cierto cambio desde el activismo político, mediante acciones disruptivas de las clásicos movimientos sociales urbanos, al activismo más moderado, centrado en la presentación de propuestas y la colaboración en la prestación de servicios, como resultado de los cambios en la agenda de actuación municipal y la progresiva extensión del Estado del bienestar (Urrutia, 1992; Navarro, 2001); lo que describiría cierta evolución entre diferentes funciones asociativas.

${ }^{2}$ El estudio ha sido financiado por el Plan Nacional de I+D+I y el Instituto de la Mujer (Convocatoria 2006) y ha consistido en un estudio comparado de casos, en el que se han seleccionado cinco municipios españoles mayores de 50.000 habitantes (Córdoba, Sevilla, Granada, Madrid y Barcelona), en los que se ha llevado a cabo una encuesta a asociaciones de cada municipio. 
A este respecto, es sabido que las asociaciones, entre otras cuestiones, se diferencian por cierta especialización respecto al hecho de que sus funciones se orienten hacia dos polos: por un lado, la prestación de servicios, ya sea a través de actividades destinadas a los socios y sociales o a través de prestación de servicios a la ciudadanía en general. Y por otro lado, la transmisión de demandas, usando para ello distintos repertorios de acción, desde la mediación mediante contactos informales o la participación en diversos organismos y procesos de participación, hasta la protesta mediante el desarrollo de actividades de carácter disruptivo, como concentraciones, manifestaciones o la movilización social a través de los medios de comunicación. De ambas funciones son las primeras las que se orientan principalmente y cabe esperar una mayor implicación en los acuerdos con la Administración para la prestación de servicios públicos, tal y como han mostrado otros análisis para el caso español (Morales y Mota, 2006)

Así, desde una perspectiva histórica, ello supondría que la extensión de los servicios personales, como los servicios de bienestar en general, han favorecido la incentivación de la función de prestación de servicios sobre la de carácter político; esto es, un cambio en las ecologías asociativas municipales y sus patrones de interacción con las administraciones, tal y como sostienen Navarro (2002). Pero también, entre las ecologías asociativas en un momento determinado existen diferencias en el grado de implicación en los procesos de coproducción en función de su especialización funcional hacia la prestación de servicios, delimitando espacios y actores diferenciados para la cooperación en la provisión y la coproducción (Navarro y Juaristi, 2006).

Desde esta última perspectiva, que supone la comparación de distintas asociaciones en un mismo momento, también puede ser importante la consideración de los recursos con los que cuenta la asociación, no sólo de carácter económico, sino de información y organización, como los que puedan provenir de su pertenencia a redes, plataformas, federaciones o como partes de organizaciones más amplias. Así, esta cobertura organizativa proporcionaría a las asociaciones de recursos organizativos y les facilitaría su implicación en los procesos de coproducción de servicios, como por ejemplo: el desarrollo de pro- yectos del personal técnico, el apoyo financiero, las experiencias realizadas en otros lugares o el apoyo técnico en caso necesario. De hecho, son las grandes entidades asociativas las que suelen establecer este tipo de procesos de colaboración con las administraciones públicas, o al menos, son los procesos más conocidos.

En lo que se refiere a los efectos de la coproducción en la dinámica organizativa de las asociaciones, suelen señalarse principalmente dos ideas: por un lado, aquella que supone la profesionalización y el aumento de la complejidad organizativa de las asociaciones; y por otro lado, que esa burocratización implica una menor participación activa y dinámica democrática en el seno de las asociaciones. El hecho de tener que prestar servicios implicaría que la asociación, como organización, debe dotarse del personal, estructura y procesos organizativos adecuados para ello, lo que pasaría por un mayor grado de profesionalización al tener que contar con personal técnico para la planificación y ejecución de los servicios; así como una mayor complejidad organizativa, y con ello, un mayor distanciamiento y menores posibilidades de participación de sus miembros en la toma de decisiones. En este sentido, algunos análisis muestran esta relación en las asociaciones españolas (Méndez y Mota, 2006)

Un aspecto menos analizado de los procesos de coproducción se refiere a su contenido; esto es, a qué tipo de servicios se prestan y para qué tipo de colectivos (o de necesidades). Evidentemente, a este respecto cabría esperar bastante diversidad, la que se corresponde con los sectores o ámbitos de actuación específicos de cada asociación o sectores asociaciones, tal y como revelan otros análisis para el caso español (Morales y Mota, 2006). Ahora bien, también cabría pensar que existe cierta diferencia o especialización en función del nivel administrativo con el que se establecen los acuerdos, dada las distintas competencias que tienen respecto a diferentes ámbitos de política pública (Rodríguez y Navarro, 2009; Gutiérrez y Garcés, 2000; Gutiérrez, 2001).

\section{El análisis de la coproducción asociativa: preguntas y metodología}

De lo expuesto anteriormente, derivan tres preguntas sobre las que trataremos de aportar evidencias en este trabajo: ¿cuántas y qué asocia- 
ciones se involucran en procesos de coproducción de servicios públicos?, ¿cuál es el contenido de los servicios según el nivel administrativo con el que establecen los acuerdos? y la coproducción ¿afecta a la dinámica organizativa de las asociaciones?

Para dar respuesta a las preguntas planteadas utilizaremos una encuesta, realizada entre asociaciones de cinco municipios españoles en el marco del estudio Género, Participación y Sistemas Locales de Bienestar. Se trata de una encuesta a asociaciones del estudio GPySLB (CSPL, 2009). El cuestionario se ha administrado a través de entrevistas personales y telefónicas a miembros de las Juntas Directivas de las asociaciones, que se distribuyen de la siguiente manera: presidente/a (54,1 por ciento), vicepresidentes/as (8,9 por ciento), tesoreros/as $(4,2$ por ciento), secretarios/as (17,7 por ciento), vocales ( 15,1 por ciento), técnicos/as $(0,9$ por ciento). En concreto, el cuestionario se aplicó a los tres tipos básicos de asociaciones que la literatura señala: político-institucionales, de prestación de servicios de bienestar y el orientado a los servicios socio-culturales y de ocio (Montero, Font y Torcal, 2006). Así, la muestra se centró en tres tipos concretos de asociaciones que representan bastante bien estas tres grandes categorías; a saber: asociaciones vecinales, asociaciones de acción social y asociaciones culturales. Además se ha incluido una muestra de asociaciones de mujeres (Anexos 1 y 2), porque representan un sector asociativo emergente, aunque minoritario, que se caracteriza por cierto pluralismo interno en el que pueden identificarse, al menos, dos tipos: las asociaciones de mujeres feministas, de carácter político y reivindicativo, y las asociaciones de mujeres orientadas a la prestación de servicios (Murillo, 2003; Valiente, 1999). A partir de los listados o censos de asociaciones municipales se elaboró una muestra representativa para cada sector, habiendo ponderado la muestra posteriormente por ciudad y sector asociativo. En total, las respuestas obtenidas han sido un total de 1.012 asociaciones, lo que supone un error del 4 por ciento para el conjunto de la muestra, bajo supuesto de máxima variabilidad y un nivel de confianza del 95 por ciento.

Para medir la extensión de la coproducción, se les preguntó a las asociaciones si mantenían un convenio, acuerdo o contrato para la presta- ción de servicios de titularidad pública (La pregunta formulada era: En concreto, durante los últimos dos años, ¿ha firmado un convenio, acuerdo o contrato para prestar un servicio de titularidad pública? Además para conocer el perfil de las asociaciones que se involucran en estos procesos y los factores que lo explican se han construido 6 indicadores relacionados con la mayor o menor predisposición de las asociaciones a celebrar ese tipo de convenios, acuerdos o contratos.

En primer lugar, atendiendo a lo planteado anteriormente, era de esperar que las asociaciones más orientadas a los servicios fueran las que más tendencia mostrasen a realizar acuerdos de colaboración para prestar servicios de titularidad pública. Por ello, el primer conjunto de indicadores, elaborados a partir de las principales actividades a las que las asociaciones declaraban haberse dedicado durante el último año, ha pretendido medir esta dimensión a través de cuatro indicadores específicos: 1) la orientación hacia el activismo político de mediación, 2) la orientación al activismo político de protesta, 3) la orientación a la prestación de servicios socio-culturales y de ocio y 4) la orientación a la prestación de servicios profesionales. Las respuestas se han agrupado del siguiente modo: 1) activismo político de mediación (reuniones y contactos con autoridades, participar en consejos consultivos, dirigir escritos a las autoridades), 2) activismo político o de protesta (conferencias de prensa/envío de comunicados, recogida de firmas/reparto de octavillas, convocar concentraciones/manifestaciones), 3) activismo de servicios socio-culturales (actividades recreativas, culturales y/o deportivas, actividades de acción social) y 4) activismo de servicios profesionales (talleres y cursos, actividades y talleres para fomentar igualdad, servicios profesionales). Para cada indicador las respuestas se han transformado en escala $(0,1)$.

En segundo lugar, el hecho de que pertenecieran a una plataforma o federación o si el ámbito territorial de actuación de la asociación era estrictamente local (el municipio o una zona/barrio de éste) o más amplio, ya que en ambos casos podía dar cuenta de la disposición de recursos y apoyos organizativos que facilitarían su implicación en los procesos de coproducción de servicios públicos. Todos estos indica- 
dores han sido estandarizados en escalas $[0,1]$ para facilitar una interpretación conjunta de los mismos, en los que el valor cero indicaba el nivel mínimo del indicador y el valor uno su nivel máximo; o bien, la ausencia frente a la presencia de la característica que se tratase.

Con respecto a la segunda cuestión planteada, el contenido de la co-producción (con quien, qué y para quién), se preguntó a las asociaciones - a través de preguntas abiertas - por el organismo con el que han establecido acuerdo de colaboración, el tipo de servicio para el que lo establecen y la población destinataria de dichos servicios; pudiendo dar, en cada una de ellas, tres respuestas. Respecto al primer aspecto, las respuestas se han codificado, considerando cuatro categorías según el nivel de gobierno con el que existen acuerdos de colaboración: Administración local (que comprende ayuntamientos, mancomunidades y diputaciones, aunque son los primeros la categoría más frecuente), Administración autonómica, Administración central y administración multinivel provincial. Respecto al «tipo de servicio», las respuestas se han clasificado en tres grandes categorías: la provisión de un bien o un servicio específico que supone la atención a necesidades o colectivos de forma especializada (atención en centros y unidades de estancia diurna, atención integral a mujeres víctimas de violencia de género, ayuda a domicilio, atención a personas con daño cerebral, atención temprana a niños con discapacidad intelectual), la provisión de recursos de carácter formativos (cursos, talleres, etc.) o la facilitación de información o asesoramiento (jurídico, psicológico, etc.). Por último, respecto al sector al que se orientan los servicios, las respuestas se han clasificado en dos grandes grupos: población en general frente a sectores específicos. Además, se han detallado los sectores siguientes: exclusión social (personas sin hogar, adicciones, prostitución, minorías, desempleados, etc.), inmigración, discapacidad y gran dependencia (mayores) y mujer.

En cuanto a la tercera cuestión, referida a los posibles efectos de la coproducción en las asociaciones, se han considerado tres indicadores; por un lado, en relación a la profesionalización se ha calculado el peso de la plantilla remunerada de la asociación calculada sobre el total de personas que la componen. Respecto a la estructura organizativa se ha considerado la existencia de seis tipos de cargos y organización: presidente o presidenta, secretario o secretaria, tesorero o tesorera; consejo directivo, comisiones específicas para tareas concretas y asamblea general. La gran mayoría desempeña los cuatro primeros cargos, alrededor del 80 por ciento y declaran tener consejo directivo, y tan sólo algo más del 50 por ciento se organiza en comisiones temáticas específicas. Así, en el indicador de complejidad organizativa elaborado como sumatorio de estos seis órganos, las que alcanzan niveles más altos se debe, fundamentalmente, a su diversificación organizativa; esto es, a que cuentan con comisiones sectoriales de trabajo.

Otra dimensión de las asociaciones que puede verse afectada por los procesos de coproducción es el de los procesos de democracia interna. Para medir este aspecto se ha construido el indicador de democracia interna, a partir de la frecuencia en la que se realizan asambleas generales en las asociaciones.

Para responder a las tres preguntas planteadas hemos hecho análisis diferentes. Así, para saber qué asociaciones suelen involucrarse en los procesos de coproducción hemos realizado análisis de regresión logística, incluyendo los indicadores de activismo asociativo, la pertenencia a una federación y el ámbito de actuación. Se espera que las asociaciones que se orientan en mayor medida al activismo de servicios profesionales son las que destacan, y en menor medida, si pertenecen a una federación o su ámbito de actuación no se reduce al ámbito local.

El análisis del contenido de los servicios que se coproducen se centra en el peso que tienen diferentes administraciones, los tipos de servicios y la población a la que se orientan, aunque también consideraremos si la Administración a la que se orientan influye en los otros dos aspectos, en la medida en que tienen en general competencias y ámbitos territoriales de actuación diferenciados.

Por último, para conocer si la coproducción influye en la profesionalización y complejidad organizativa de las asociaciones hemos realizado un análisis de diferencias de medias, pero también modelos de regresión para esas dos variables, incluyendo como variables independientes, además de la coproducción, la importancia de diferentes tipos de activismo, la per- 
tenencia a federaciones, el ámbito de actuación y el porcentaje de su presupuesto que proviene de administraciones públicas. Con ello se pretende conocer si, una vez controlado el efecto de estas variables, la coproducción influye en la profesionalización y complejidad organizativa de las asociaciones. Evidentemente, tanto en este caso como en el análisis referido a la extensión de la colaboración, no cabe derivar una especificación precisa de relación causal, en la medida en que para ello sería necesario un diseño que considerase el cambio en la especialización funcional o la estructura organizativa de las asociaciones antes y después de establecer un acuerdo para la coproducción de servicios públicos. No obstante, los análisis permitirán señalar si existen asociaciones significativas y consistentes que avalen los argumentos presentados más arriba.

\section{Análisis y resultados}

A continuación presentamos los resultados de los análisis realizados, así como su discusión, en el orden de las preguntas previamente planteadas. En primer lugar, la extensión del fenómeno de la coproducción y los rasgos de las asociaciones que se involucran en la misma.
En segundo lugar, el contenido de esos procesos, y en particular, si la esfera de la Administración Pública con la que establecen acuerdos para la coproducción de servicios influye en el tipo de servicios que se prestan (su naturaleza y el sector de población al que van dirigidos). Por último, si la coproducción implica cambios en la dinámica y estructura organizativa de las asociaciones, en términos de: profesionalización, complejidad organizativa y democracia interna.

\subsection{La extensión de la coproducción: la es- pecialización del activismo asociativo}

Del total de asociaciones entrevistadas, el 19,6 por ciento declara haber firmado un convenio, acuerdo o contrato para prestar un servicio de titularidad pública (calculado de respuesta a la pregunta: En concreto, durante los últimos dos años, ¿ha firmado un convenio, acuerdo o contrato para prestar un servicio de titularidad pública?).

Si consideramos los tipos asociativos incluidos en el estudio, el 23,6 por ciento corresponde al tipo asociativo «acción social», que - como se ha dicho - representa al sector de asociaciones que destacan por orientarse a la

\begin{tabular}{|c|c|c|c|}
\hline $\begin{array}{c}\text { Preguntas } \\
\text { sobre la coproducción }\end{array}$ & Variables dependientes & $\begin{array}{l}\text { Posibles variables explicativas } \\
\text { (incluidas en los análisis) }\end{array}$ & $\begin{array}{l}\text { Posibles respuestas } \\
\text { (variable explicativa } \\
\text { más importante) }\end{array}$ \\
\hline $\begin{array}{l}\text { Extensión: } \\
\text { ¿Quiénes coproducen? }\end{array}$ & Coproducción & $\begin{array}{l}\text { Activismo: político } \\
\text { y de servicios } \\
\text { Pertenencia a una } \\
\text { federación } \\
\text { Ámbito: local vs. no } \\
\text { local }\end{array}$ & $\begin{array}{l}\text { Activismo de servicios } \\
\text { profesionales }\end{array}$ \\
\hline $\begin{array}{l}\text { Contenidos: } \\
\text { ¿Influye el nivel } \\
\text { administrativo en el } \\
\text { contenido de la } \\
\text { coproducción? }\end{array}$ & $\begin{array}{l}\text { Tipos de servicios } \\
\text { Población en general } \\
\text { vs. sectores específicos }\end{array}$ & Nivel administrativo & $\begin{array}{l}\text { Administración local: } \\
\text { servicios a población } \\
\text { en general centrados } \\
\text { en el asesoramiento } \\
\text { y la formación }\end{array}$ \\
\hline $\begin{array}{l}\text { Consecuencias: } \\
\text { ¿Se profesionalizan? }\end{array}$ & $\begin{array}{l}\text { Profesionalización } \\
\text { Complejidad orgnizativa } \\
\text { Democracia interna }\end{array}$ & $\begin{array}{l}\text { Activismo: político } \\
\text { y de servicios } \\
\text { Pertenencia a una } \\
\text { federación } \\
\text { Ámbito local vs. no } \\
\text { local } \\
\text { \% financiación pública }\end{array}$ & $\begin{array}{l}\text { Sí, con independencia } \\
\text { de los otros factores }\end{array}$ \\
\hline
\end{tabular}

Tabla 1. Asociaciones y coproducción de servicios públicos: algunas preguntas y posibles respuestas. 


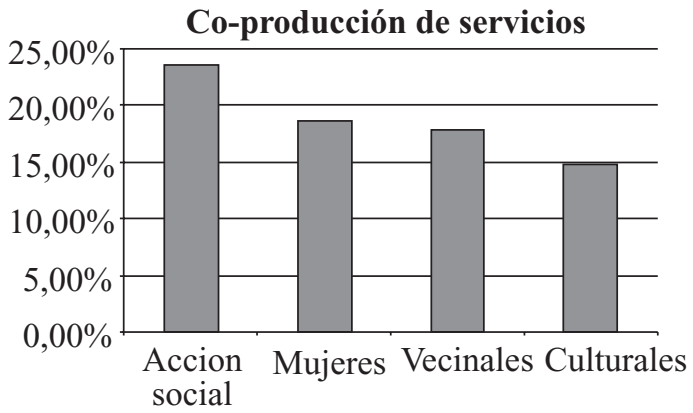

Gráfico 1. Coproducción según sectores asociativos. Porcentaje sobre total de asociaciones en cada sector asociativo.

Fuente: Encuesta asociaciones del estudio GPySLB (CSPL, 2009).

prestación de servicios. Los otros tipos asociativos presentan niveles más bajos, desde el 18,6 por ciento entre las asociaciones de mujeres, hasta el 17,7 por ciento de las asociaciones vecinales y el 14,8 por ciento de las asociaciones culturales y de ocio. Así y como cabría esperar, parece existir bastante diferencia entre aquellos sectores asociativos que se orientan principalmente a la prestación de servicios y los demás, entre los cuales existe una mayor heterogeneidad al respecto.

Por lo tanto, razonable que por su naturaleza sean las asociaciones de «acción social» quienes establezcan más acuerdos de colaboración con la Administración Pública. Sin embargo, cabe destacar que entre los otros sectores asociativos también existe una tendencia, aunque menos destacada, hacia la coproducción de servicios. De hecho, la literatura sobre el pluralismo de las asociaciones de mujeres destaca que en ellas existe una diferenciación entre aquellas más orientadas a los servicios y las que lo hacen a la transmisión de demandas (Ortbals, 2009). Por otro lado, aunque las de carácter cultural y recreativo suelan centrarse en la provisión de servicios a sus miembros, no implica que para ello no establezcan acuerdos de colaboración con la Administración Pública. Y por último, aunque las asociaciones vecinales son, de los tipos considerados, las que más destacan por su especialización política, como movimientos sociales urbanos, bien es cierto que desde los años ochenta han venido cambiando para acercarse más hacia actividades que implican la mediación y el contacto con la Administración a través de distintos órganos de participación, así como la participación en la prestación de algunos servicios urbanos básicos (Urrutia, 1992; Navarro, 2001). Así, pues, en todos estos sectores cabría establecer diferencias, más o menos marcadas a lo largo de la dimensión «prestación de servicios versus activismo político».

Tal y como puede apreciarse en la Tabla 2, que muestra los resultados de análisis de regresión logística con las variables explicativas consideradas para analizar al extensión de la coproducción, son las relativas a la especialización en algunas actividades las que en mayor media dan cuenta del fenómeno. Aunque las tendencias en las otras variables consideradas apuntan a lo que pudiera considerarse previsible (mayor coproducción entre las integradas en una federación, con ámbito de actuación más amplio que el local, orientadas a los servicios de ocio, pero no al activismo de protesta), sus coeficientes no son significativos (para $p<0,05$ ). Por lo tanto, cabría señalar que el fenómeno de

\begin{tabular}{|l|r|}
\hline \multicolumn{1}{|c|}{ Variables dependientes } & \multicolumn{1}{c|}{ B (error) } \\
\hline Funciones & $\mathbf{1 , 1 3 1}(\mathbf{0 , 3 7 6 )}$ \\
Activismo político: mediación & $-0,353(0,275)$ \\
Activismo político: protesta & $0,281(0,270)$ \\
Activismo de servicios: culturales y de ocio & $\mathbf{1 , 2 1 1}(\mathbf{0 , 2 8 6})$ \\
Activismo de servicios: profesionales & $0,167(0,178)$ \\
\hline Pertenencia a federación (sí) & $-0,243(0,169)$ \\
\hline Ámbito de actuación (local) & $\mathbf{- 3 , 0 0 4}(\mathbf{0 , 3 4 4})$ \\
\hline Constante & $\mathbf{0 , 0 8 0}$ \\
\hline R2 (Nagelkerke) & \\
\hline
\end{tabular}

Tabla 2. La coproducción según funciones y cobertura organizativa de las asociaciones. 
la coproducción se relaciona fundamentalmente con el hecho de desarrollar un activismo político no invasivo, aquel centrado en el contacto informal con autoridades públicas para transmitir demandas o la presencia en organismos de participación, y la especialización en la provisión de servicios de carácter profesional; esto es, aquellos que se orientan a la atención de cuestiones relacionados con la existencia de desigualdades respecto al bienestar de la población o grupos específicos. Se trata de resultados que vienen a confirmar otros realizados en España (Navarro y Juaristi, 2006), o en general, las ideas mayoritarias en la literatura al respecto. Aunque es de destacar, que no sólo se trata de especialización en la producción de servicios, sino que en ello también cuenta la mediación política. Esto es, son asociaciones que no permanecen aisladas de su entono político, que tratan de transmitir demandas a las autoridades públicas, ahora bien, mediante repertorios que suponen procesos de contacto informal o la canalización a través de organismos y procesos participativos, no mediante el activismo político disruptivo.

Evidentemente con este análisis de corte transversal no podemos establecer claramente una relación causal, pero al menos podemos concluir que existe cierta asociación entre la especialización funcional de las asociaciones y el hecho de que se involucren en procesos de coproducción de servicios públicos; concretamente, esto último parece ligarse a la especialización en la producción de servicios y el uso de repertorios de activismo político más moderados.

\subsection{El contenido de la coproducción: qué, con quién, para quién}

Pero, ¿cuál es el contenido de la coproducción? ¿con quién se establecen los acuerdos? ¿hacia qué grupos y demandas se orientan los servicios que son objeto de la co-producción? Al tratarse de preguntas de respuestas múltiple y al centrarse nuestro interés en las actividades que realizan las asociaciones, los porcentajes que se presentan en esta sección se basan en el total de respuestas (no en el total de casos, de asociaciones). Evidentemente, el análisis se circunscribe únicamente a aquellas asociaciones que declararon que habían firmado un acuerdo para prestar un servicio de titularidad pública.
Con respecto a la primera cuestión, el 53,4 por ciento de los acuerdos que declaran las asociaciones corresponden a la atención a necesidades y colectivos, esto es la provisión de un bien concreto.

Las respuestas restantes se distribuyen de forma equilibrada entre las otras dos categorías: la provisión de recursos formativos $(26,6$ por ciento) y el asesoramiento o información (20,0 por ciento). Si se atiende al sector de población o a problemas a los que se orientan los servicios cabe destacar que sobre el 35 por ciento lo hacen hacia la población en general. No obstante, cabe destacar la existencia de grupos específicos que presentan necesidades especiales. Así, por orden de importancia, el 20,7 por ciento se orientan a la atención a la discapacidad o la gran dependencia (mayores), el 19,9 por ciento a problemas o a personas en situación o riesgo de exclusión, el 13,6 por ciento a la infancia y la juventud, el 6,20 por ciento se orienta a la mujer, y por último, el 4,8 por ciento lo hace respecto a la inmigración.

Por último, respecto al organismo con el que se establecen los acuerdos, destaca claramente la Administración autonómica, que acumula casi la mayoría de las respuestas recabadas $(58,5$ por ciento). Le sigue la Administración Local (33,5 por ciento), siendo menores al 10 por ciento las otras dos categorías de respuesta: la Administración central y aquella que supone la participación conjunta de varios niveles de gobierno. En resumen, se trata de servicios que suponen principalmente la provisión de bienes o la capacitación a la población en general o que presenta cierto riesgo de exclusión en general, o en particular a la discapacidad; especialmente, se trata de acuerdos con niveles subnacionales, y con el autonómico en particular. Este seria el perfil modal de los acuerdos de colaboración que declaran las asociaciones en la encuesta, tal y como puede apreciarse en el Gráfico 2.

Pero, ¿existe alguna relación entre el nivel de gobierno con el que se establecen los acuerdos y el tipo de servicios que se coproducen? En la Tabla 3 puede apreciarse que cuando se trata de acuerdos con las administraciones autonómicas y la central, o en los que estas intervienen, los servicios que se coproducen son los de atención específica: alrededor de un 60 por ciento de los acuerdos que se establecen con la 


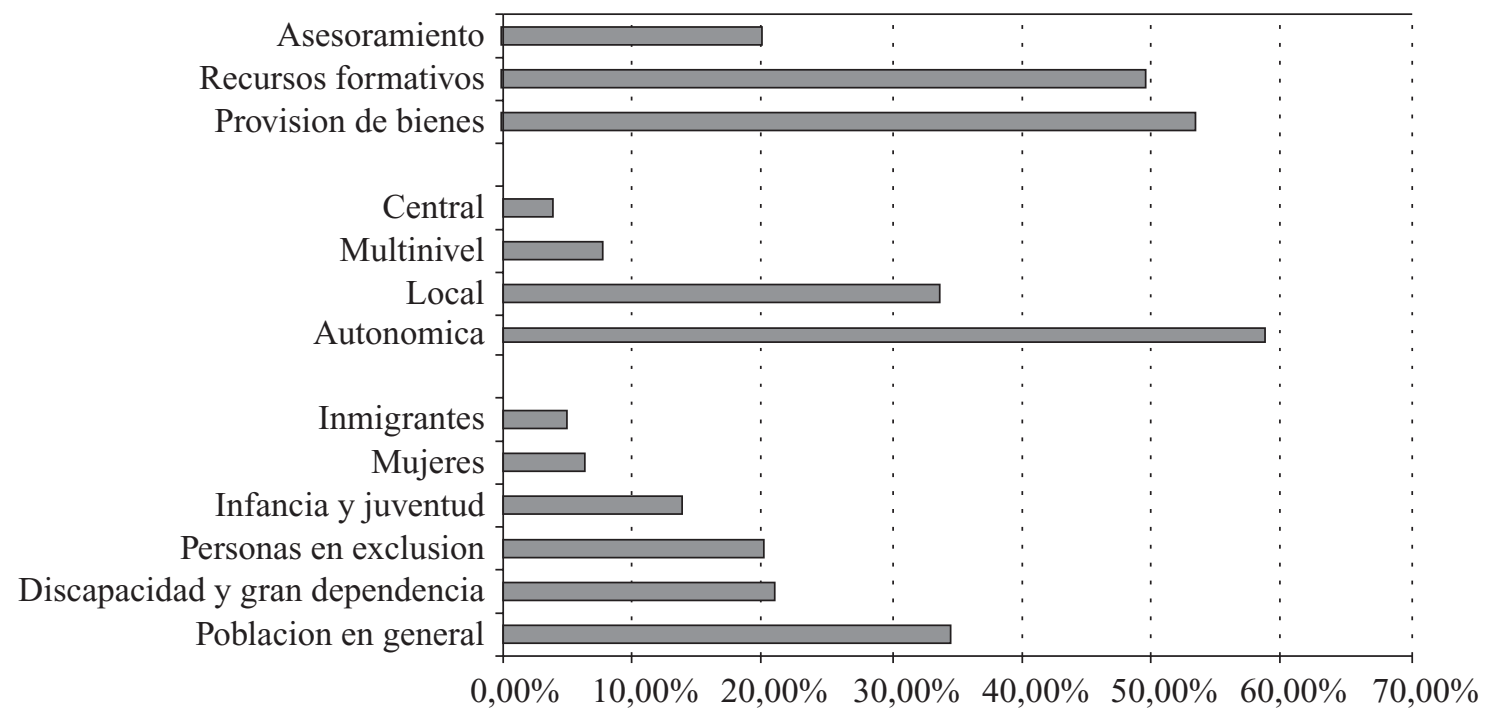

Gráfico 2. El contenido de la coproducción: tipo, sector y nivel administrativo. Porcentajes sobre el total de respuestas (servicios).

Fuente: Encuesta asociaciones del estudio GPySLB (CSPL, 2009).

Administración autonómica y central, y el 45 por ciento aproximadamente con las agrupaciones de distintas administraciones. Cuando se trata de acuerdos con la Administración local destaca sobre todo, la prestación de servicios relacionados con la capacitación mediante recursos formativos, tratándose principalmente de talleres destinados a ciudadanos y ciudadanas del municipio. Por último, el tipo de administración no parece influir en el hecho de que se presten servicios relacionados con actividades de apoyo y asesoramiento, en todas ellas se sitúan en niveles cercanos al 25 por ciento, con el máximo para aquellos servicios en los que intervienen varias administraciones (29 por cien- to) y el mínimo cuando se trata de la Administración autonómica (18 por ciento). Esto es, en el ámbito local se trata, fundamentalmente, de servicios de capacitación, mientras que a nivel supramunicipal, se centran en la prestación de servicios específicos; entre los cuales son mayoritarios los acuerdos que se establecen con las comunidades autónomas.

Además, también pueden apreciarse diferencias según el sector al que se orientan los servicios (Tabla 4). Así, puede verse que en el caso de la Administración local destacan los servicios orientados a la población en general (41 por ciento), y cuando se trata de sectores específicos, lo hacen los orientados a personas

\begin{tabular}{|l|c|c|c|c|}
\hline \multirow{2}{*}{ Nivel administrativo } & \multicolumn{3}{|c|}{ Tipo de servicio } & \multirow{2}{*}{ Total } \\
\cline { 2 - 4 } & Asesoramiento & Provisión de bienes & Recursos formativos & \\
\hline Local & 26,6 & 32,8 & 40,6 & $100,0(155)$ \\
\hline Autonómica & 18,3 & 60,1 & 21,6 & $100,0(390)$ \\
\hline Central & 26,4 & 63,6 & 10,0 & $100,0(23)$ \\
\hline Multinivel & 29,0 & 44,9 & 26,1 & $100,0(24)$ \\
\hline Total & 20 & 53,4 & 26,6 & $100,0(592)$ \\
\hline
\end{tabular}

Tabla 3. Tipo de servicio y niveles de gobierno. Porcentajes sobre el total de acuerdos establecidos con cada Administración.

Fuente: Encuesta asociaciones del estudio GPySLB (CSPL, 2009). 


\begin{tabular}{|l|c|c|c|l|}
\hline $\begin{array}{c}\text { Nivel } \\
\text { administrativo }\end{array}$ & $\begin{array}{c}\text { Población } \\
\text { en general }\end{array}$ & $\begin{array}{c}\text { Sectores } \\
\text { específcos }\end{array}$ & \multicolumn{1}{|c|}{ Total } & \multicolumn{1}{|c|}{ Los dos sectores más importantes } \\
\hline Local & 51,7 & 48,3 & $100,0(219)$ & $\begin{array}{l}\text { Exclusión (19\%) } \\
\text { Inmigración (9\%) }\end{array}$ \\
\hline Autonómica & 23,9 & 76,1 & $100,0(422)$ & $\begin{array}{l}\text { Discapacidad y gran dependen- } \\
\text { cia (31\%) } \\
\text { Exclusión (22\%) }\end{array}$ \\
\hline Central & 21,4 & 78,6 & $100,0(35)$ & $\begin{array}{l}\text { Infancia y juventud (22\%) } \\
\text { Mujer (16\%) }\end{array}$ \\
\hline Multinivel & 46,3 & 53,7 & $100,0(33)$ & $\begin{array}{l}\text { Exclusión (19\%) } \\
\text { Infancia y juventud (13\%) }\end{array}$ \\
\hline Total & 35,8 & 64,2 & $100,0(709)$ & \\
\hline
\end{tabular}

Tabla 4. Sectores y nivel administrativo. Porcentajes sobre el total de acuerdos en cada nivel administrativo. Fuente: Encuesta asociaciones del estudio GPySLB (CLPL, 2009).

en situación o riesgo de exclusión (19 por ciento) o inmigrantes ( 9 por ciento). En los otros ámbitos administrativos destacan, en cambio, servicios orientados a sectores específicos. En concreto, los relativos a exclusión social para el ámbito autonómico (22 por ciento), y sobre todo, los de discapacidad y gran dependencia ( 31 por ciento), infancia y juventud y mujer en el ámbito estatal (22 por ciento y 16 por ciento, respectivamente); aunque, en todo caso, se trata de un número bastante más reducido de respuestas (servicios).

Como es obvio, los sectores que aparecen se relacionan con los tipos asociativos incluidos en el estudio, pero también pueden apreciarse diferencias relevantes: desde la población general para los municipios, la exclusión social en las comunidades autónomas o grupos específicos en el ámbito estatal. En todo caso, la diferencia más importante es la que atañe a la distinción entre servicios orientados a la población en general y los orientados a colectivos o sectores específicos, en donde destaca la comunidad autónoma, con quien las asociaciones establecen acuerdos de forma mayoritaria.

\subsection{Los efectos de la coproducción en las asociaciones: profesionalización, complejidad} organizativa y democracia interna

Tal y como se ha dicho antes en uno de los aspectos en que se centra en mayor medida el debate sobre la coproducción de servicios públicos por parte de las asociaciones es el de cómo les repercute la misma, aunque en general no suela analizarse empíricamente. Aquí nos centraremos en tres aspectos que suele mencionar la literatura: complejidad organizativa, profesionalización y democracia interna. ¿Existen diferencias entre las asociaciones que se involucran en procesos de coproducción y las que no lo hacen?

Tal y como puede apreciarse en el Gráfico 3, la respuesta es afirmativa: existen diferencias significativas entre las asociaciones que coproducen y las que no coproducen servicios: las primeras alcanzan niveles más altos de profesionalización, complejidad organizativa y democracia interna, como frecuencia de reuniones de sus asambleas. Esto es, su implicación en la prestación de servicios públicos implica una mayor presencia de técnicos (personal contratado), una mayor diversificación de su organización, y un mayor control de sus miembros sobre las actividades asociativas; cabría decir que no existe un menor activismo político interno en las asociaciones, sino más bien lo contrario; lo que puede interpretarse como un mayor ejercicio de control democrático sobre sus actividades.

Evidentemente, estas diferencias en la dinámica y estructura organizativas pueden derivarse de la propia especialización funcional de las asociaciones e incluso porque reciban más financiación pública, sea para prestar servicios, sea para el desarrollo de sus actividades. ¿Existe un efecto de la implicación en la coproduc- 


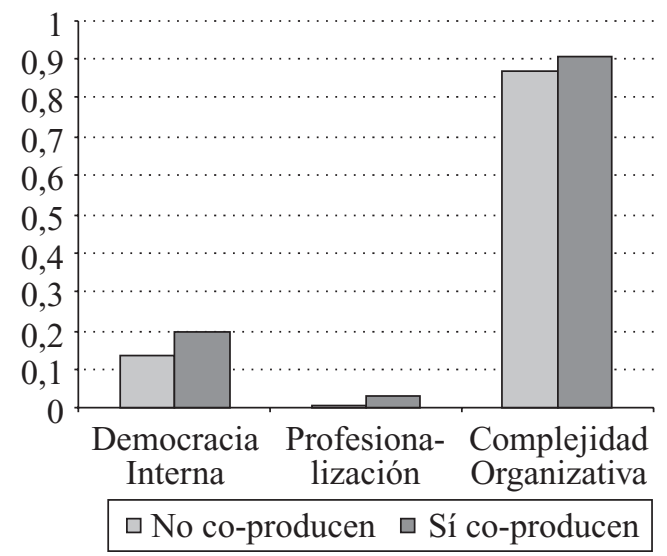

Gráfico 3. Las consecuencias de la coproducción: profesionalización, complejidad organizativa y frecuencia reuniones de la asamblea de socios. Media en escalas $(0,1)$.

Nota: Todas las diferencias son significativas $(\mathrm{p}<0,05)$. Fuente: Encuesta asociaciones del estudio GPySLB (CSPL, 2009).

ción de servicios con independencia de estos factores? Los análisis de regresión que se muestran en la Tabla 5 lo evidencian: la coproducción implica los cambios organizativos señalados con independencia de su especialización funcional, cobertura organizativa o el peso de la financiación pública en sus presupuestos. Es cierto que una mayor financiación pública y la especialización en el activismo de servicios incrementan los niveles de profesionalización, pero ello no significa que no exista el efecto de la coproducción. Del mismo modo, el efecto persiste en lo que se refiere a la complejidad organizativa, aunque en ello influya también la especialización en prestación de servicios.

Por último, también influye la coproducción en la frecuencia de las reuniones de la asamblea, junto a la especialización, tanto de servicios, como de mediación, sin que en ello cuente el porcentaje o la dependencia de la financiación pública. Como en análisis anteriores, no podemos señalar que exista una clara relación causal, pero sí que la coproducción se asocia a los cambios organizativos con cierta independencia de otras características asociativas que pueden tener relación con ellos.

\section{Conclusiones}

En términos generales, las evidencias mostradas en el apartado anterior vienen a confirmar algunas de las ideas presentadas sobre la coproducción de servicios públicos por parte de las asociaciones. Concretamente en sentido afirmativo cabe destacar la relación entre la especialización funcional en la prestación de servicios y la extensión de la coproducción, o sus efectos en un mayor grado de complejidad organizativa y profesionalización.

En cambio no supone una menor dinámica participativa en las asociaciones, cuando menos en lo que se refiere a la frecuencia con la que se reúne la asamblea. No tenemos información sobre los miembros de las asociaciones, pero este indicador, al menos, señala que la coproducción supone una mayor frecuencia de con-

\begin{tabular}{|l|c|c|c|}
\hline & Profesionalización & $\begin{array}{c}\text { Complejidad } \\
\text { organizativa }\end{array}$ & $\begin{array}{c}\text { Frecuencia } \\
\text { reuniones } \\
\text { asamblaea }\end{array}$ \\
\hline Activismo político: mediación & B (error tip.) & B (error tip.) & B (error tip.) \\
\hline Activismo servicios: profesionales & $-0,007(0,005)$ & $0,106(014)$ & $0,014(0,034)$ \\
\hline Federación & $\mathbf{0 , 0 1 0}(\mathbf{0 , 0 0 4 )}$ & $0,033(0,013)$ & $0,004(0,030)$ \\
\hline Financiación pública & $-0,001(0,003)$ & $0,023(0,008)$ & $-0,054(0,020)$ \\
\hline Coproducción servicios & $\mathbf{0 , 0 1 8}(\mathbf{0 , 0 0 4})$ & $-0,019(0,012)$ & $0,019(0.029)$ \\
\hline R2 & $\mathbf{0 , 0 1 2}(\mathbf{0 , 0 0 4})$ & $0,029(0,011)$ & $\mathbf{0 , 0 7 0}(\mathbf{0 , 0 2 5})$ \\
\hline
\end{tabular}

Tabla 5. Coproducción y aspectos organizativos: profesionalización y complejidad. Regresiones lineales. Nota: En negrillas, coeficientes significativos para $\mathrm{p}<0,05$.

Fuente: Encuesta asociaciones del estudio CPOySLB (CSPL, 2009). 
trol democrático de los miembros sobre la asociación.

Por último, cabe destacar que es con la Administración autonómica con quien se establecen en mayor medida acuerdos de colaboración para la prestación de servicios públicos, que son de naturaleza distinta y están orientados a sectores muy diversos. En ello, sin duda, cuenta la centralidad de este nivel administrativo en la prestación de servicios personales. Más allá de estas afirmaciones, los análisis no permiten concluir claramente sobre la relación entre el nivel administrativo con el que las asociaciones establecen acuerdos y el tipo o sector para el que lo hacen.

En todo caso, existe un mayor grado de especialización y diversificación cuando se trata de niveles supramunicipales, el autonómico en particular, donde se trata de la producción de servicios concretos, porque cuando se trata del ámbito local, las asociaciones declaran que los servicios se orientan en mayor medida a la población en general y se centran más en actividades de capacitación o asesoramiento.

En parte, la menor claridad en el análisis de los contenidos de los procesos de coproducción puede deberse a los sectores asociativos incluidos en el estudio, que focalizan el ámbito, sector y tipo de servicios hacía algunas categorías de respuesta. Pero también es cierto que existe bastante diversidad entre ellos como para tomar nuestras evidencias como un primer acercamiento al asunto.

También hemos indicado que este diseño basado en una encuesta en un momento del tiempo, no permite ofrecer afirmaciones concluyentes sobre la causalidad entre las características de las asociaciones y el hecho de implicarse en acuerdos de coproducción. Para ello sería más adecuado un diseño que considerase el cambio en momentos anteriores y posteriores al establecimiento de tales acuerdos.

No obstante, ofrece asociaciones bastante claras y concordantes con propuestas analíticas y análisis previos. Por lo que, aún considerando las limitaciones que se han ido exponiendo, hemos pretendido ofrecer algunas evidencias que ayuden a la reflexión y el análisis sobre el papel de las asociaciones en el denominado welfare mix.

\section{Referencias bibliográficas}

Esping-Andersen, G. (1999). Social Foundations of Postindustrial Economies. Oxford: Oxford University Press.

Gutiérrez, A. y Garcés Ferrer, J. (coords.) (2001). El Plan Concertado de Prestaciones Básicas de Servicios Sociales en España (11 años de Plan Concertado). Revista Internacional de Sociología, 93, 89-130.

Gutiérrez, A. y Garcés, J. (coords.) (2000). Los Servicios Sociales en las Comunidades y Ciudades Autonomas. Valencia: Tirant lo Blanch.

Méndez, M. y Mota, F. (2006). Las características organizativas de las asociaciones en España. En J.R. Montero, J. Font y M. Torcal (eds.), Ciudadanos, asociaciones y participación en España, (pp.201-222). Madrid: CIS.

Montero, J. R., Font, J. y Torcal, M. (2006). Ciudadanos, asociaciones y participación en España. Madrid: CIS.

Morales, L. y Mota, F. (2006). El asociacionismo en España, en J.R. Montero, J. Font y M. Torcal (eds.), Ciudadanos, asociaciones y participación en España (pp. 77-104). Madrid: CIS.

Murillo, S. (2003). Ciudadanía Activa. Asociacionismo de Mujeres. Madrid, Comunidad de Madrid.

Navarro, C.J. (2001). La calidad del pluralismo del bienestar. Córdoba: Universidad de Córdoba.

Navarro, C.J. (2020). Democracia asociativa y oportunismo politico. Valencia: Tirant lo Blanch.

Navarro, C.J. y Juaristi, P. (2006). Funciones, actividades y facilitación pública de las asociaciones. En J.R. Montero, J. Font y M. Torcal (eds.), Ciudadanos, asociaciones y participación en España (pp. 223-239). Madrid: CIS.

Navarro C.J. y Rodríguez, M.J. (2009). Gobernanza Multi-nivel y sistemas locales de bienestar en España. El caso de las Diputaciones Provinciales. Valencia: Tirant lo Blanch.

Ortbals, C. (2008). Subnational Politis in Spain: New Avenues for Feminist Policymaking and Activism. Politics and Gender, 4, 93-119. 
Rodríguez Cabrero, G. (1994). Estado del bienestar y sociedad de bienestar. Revista Internacional de Sociología, 8-9, 7-29.

Rodríguez Cabrero, G. y Codorniú, M. (1996). Las entidades voluntarias en la construcción del bienestar social. En Las entidades voluntarias en España. Institucionalización, estructura económica y desarrollo asociativo (pp. 17-33). Madrid: MAS.

Rodríguez Cabrero, G. (2004). El estado del bienestar en España: debates, desarrollos y retos. Madrid: Fundamentos.

Rodríguez Cabrero, G. (2005). Los retos del tercer sector en España en el espacio social europeo. Revista Española del Tercer Sector, 1, 63-91.

Salamon, L. y Anheier, H.K. (1992). In search of the nonprofit sector : the question of definitions. Voluntas, 3 (2), 125-151.

Sharp, E. (1980). Towards a New Understanding of Urban Services and Participation: The Coproduction Concept. Midwest Review of Public Administration, 14, 105-118.

Urruria, V. (1992). Transformación y persistencia de los movimientos sociales urbanos, Política y Sociedad, 10, 49-56.

Valiente, C. (1999). Feminismo de Estado en los ayuntamientos de la Comunidad Autónoma de Madrid, Gestión y Análisis de Políticas Públicas, 13-14, 173-189.

Whitaker, G. (1980). Coproduction: Citizen Participation in Service Delivery. Public Administration Review, 40, 240-246.

ANEXOS

\begin{tabular}{|c|c|c|c|c|}
\hline & \multicolumn{4}{|c|}{ Ecología asociativa municipai } \\
\hline Sectores asociativos & Mujer & $\begin{array}{c}\text { Político- } \\
\text { institucional }\end{array}$ & Bienestar social & $\begin{array}{l}\text { Socio-cultural } \\
\text { y ocio }\end{array}$ \\
\hline Tipos correctos & Mujer & Vecinales & Acción social & Culturales \\
\hline \multirow[t]{2}{*}{$\begin{array}{l}\text { Criterios de selección } \\
\text { (afijación) }\end{array}$} & Afijación simple & \multicolumn{3}{|c|}{$\begin{array}{l}\text { Afijación proporcional del número de asociaciones } \\
\text { de estos tres tipos sobre el total de ellas existente } \\
\text { en cada ciudad }\end{array}$} \\
\hline & $\begin{array}{l}\mathrm{N}^{o} \text { fijo y mínimo de } \\
\text { asociaciones por } \\
\text { municipios }\end{array}$ & \multicolumn{3}{|c|}{$\mathrm{N}^{\mathrm{o}}$ variable de tipos de asociaciones por ciudad } \\
\hline $\begin{array}{l}\text { Tamaño muestral } \\
\text { diseñado por municipio }\end{array}$ & 100 & \multicolumn{3}{|c|}{140} \\
\hline $\begin{array}{l}\text { Tamaño muestral } \\
\text { diseñado total del estudio }\end{array}$ & 500 & \multicolumn{3}{|c|}{700} \\
\hline
\end{tabular}

Anexo 1. Diseño muestral de la encuesta a asociaciones.

\begin{tabular}{|l|c|c|c|c|}
\hline \multicolumn{1}{|c|}{ Tipos asociativos } & Población & Muestra diseñada & Muestra obtenida & Error (1) \\
\hline Asociaciones de mujeres & 610 & 469 & 256 & $6 \%$ \\
\hline $\begin{array}{l}\text { Otros tipos (Vecinales, Acción } \\
\text { Social y Culturales) }\end{array}$ & 6.394 & 731 & 756 & $5 \%$ \\
\hline Total & 6.004 & 1.200 & 1.012 & $4 \%$ \\
\hline
\end{tabular}

Anexo 2. Población y muestras de la encuesta a asociaciones.

(1) Supuesto de máxima variabilidad $(p=q), 95 \%$ confianza. 